\title{
BMJ Open European Working Time Directive and doctors' health: a systematic review of the available epidemiological evidence
}

\author{
Maria Cruz Rodriguez-Jareño, ${ }^{1,2}$ Evangelia Demou, ${ }^{3,4}$ Sergio Vargas-Prada, ${ }^{5}$ \\ Kaveh A Sanati, ${ }^{4,6}$ Alenka Škerjanc, ${ }^{7}$ Pedro G Reis, ${ }^{8}$ Ritva Helimäki-Aro, ${ }^{9}$ \\ Ewan B Macdonald, ${ }^{4}$ Consol Serra, ${ }^{1,5,10,11}$ on behalf of the UEMS Section \\ of Occupational Medicine ${ }^{12}$
}

To cite: RodriguezJareño MC, Demou E, Vargas-Prada S, et al. European Working Time Directive and doctors' health: a systematic review of the available epidemiological evidence. BMJ Open 2014;4: e004916. doi:10.1136/ bmjopen-2014-004916

- Prepublication history for this paper is available online. To view these files please visit the journal online (http://dx.doi.org/10.1136/ bmjopen-2014-004916).

Received 24 January 2014 Revised 12 June 2014 Accepted 16 June 2014

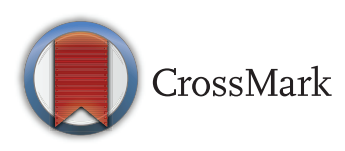

For numbered affiliations see end of article.

Correspondence to Dr Evangelia Demou; evangelia.demou@glasgow. ac.uk

\section{ABSTRACT}

Objective: To summarise the available scientific evidence on the health effects of exposure to working beyond the limit number of hours established by the European Working Time Directive (EWTD) on physicians. Design: A systematic literature search was conducted in PubMed and EMBASE. Study selection, quality appraisal and data extraction were carried out by independent pairs of researchers using pre-established criteria.

Setting: Physicians of any medical, surgical or community specialty, working in any possible setting (hospitals, primary healthcare, etc), as well as trainees, residents, junior house officers or postgraduate interns, were included.

Participants: The total number of participants was 14338.

Primary and secondary outcome measures: Health effects classified under the International Classification of Diseases (ICD-10).

Results: Over 3000 citations and 110 full articles were reviewed. From these, 11 studies of high or intermediate quality carried out in North America, Europe and Japan met the inclusion criteria. Six studies included medical residents, junior doctors or house officers and the five others included medical specialists or consultants, medical, dental, and general practitioners and hospital physicians. Evidence of an association was found between percutaneous injuries and road traffic accidents with extended long working hours (LWH)/days or very LWH/weeks. The evidence was insufficient for mood disorders and general health. No studies on other health outcomes were identified.

Conclusions: LWH could increase the risk of percutaneous injuries and road traffic accidents, and possibly other incidents at work through the same pathway. While associations are clear, the existing evidence does not allow for an established causal or 'dose-response' relationship between LWH and incidents at work, or for a threshold number of extended hours above which there is a significantly higher risk and the hours physicians could work and remain safe and healthy. Policymakers should consider safety issues when working on relaxing EWTD for doctors.

\section{Strengths and limitations of this study}

- The first systematic review, to the best of our knowledge, on the effects of long working hours (LWH) on physicians' health and safety.

- A systematic literature search conducted in PubMed and EMBASE with over 3000 citations and 110 full articles reviewed.

- Eleven studies of high or intermediate quality carried out in North America, Europe and Japan, involving physicians of any medical, surgical or community specialty, working in any possible setting (hospitals, primary healthcare, etc), as well as trainees, residents, junior house officers or postgraduate interns, were included.

- The findings of this review confirmed that LWH are associated with an increased risk of needlestick injuries and road traffic accidents. Evidence was assessed as low or insufficient for mood disorders and general health.

- This review uncovered the lack of literature on the effect of LWH on the general health of doctors, a topic that has important health ramifications not only for physicians but also for patients.

\section{INTRODUCTION}

The European Working Time Directive (EWTD),${ }^{1}$ applicable to all occupations across the European Union (EU), requires a maximum working week of $48 \mathrm{~h}$ and establishes rest periods. ${ }^{2}$ Since it came into force in healthcare in 2009, it has been associated with concerns about the provision of health services including continuity of care, lower staffing levels, introduction of shift working, a reduction in training time and the adequate supervision of junior doctors. ${ }^{4} 5$ The large intercountry variations in physicians' working hours as well as the mandatory controls over working hours across occupations raise further questions as to the 
basis of selecting and setting these limits and restrictions. ${ }^{6}$ There is evidence of variable compliance to the EWTD within healthcare across Europe. ${ }^{45}$ The medical profession is also increasingly feminised with potential implications for women of childbearing age, which may coincide with the time in the profession where long working hours $(\mathrm{LWH})$ are more likely to happen (ie, during residency). Constraint on public sector finances and increasing healthcare demands have stimulated the debate on physicians' working hours, ${ }^{7}$ with some evidence showing that the quality of healthcare is positively correlated with the number of physicians available to deliver that care. ${ }^{8}$

The Union of European Medical Specialists (UEMS) was created in 1958 to represent medical specialists in the $\mathrm{EU}$, promote a high standard of training and practice at European level and facilitate the free movement of physicians across European countries. ${ }^{9}$ The Occupational Medicine Section was created in 1997 and is involved in issues related to specialist training, professional practice and research ${ }^{10}$; elaborating and publishing reference documents, ${ }^{11}$ conducting and contributing to surveys ${ }^{12-14}$ and working in partnership with other key European groups.

The trigger for this project was a request by the Council of the UEMS to its Occupational Medicine Section for a position statement on the EWTD and possible health consequences to physicians of a potential removal of this professional group from the current EWTD $48 \mathrm{~h} /$ week limit. ${ }^{15-17}$ An evidence-based approach was taken by the Section, and a systematic review of the literature was undertaken. ${ }^{15}$

LWH have been associated with several adverse effects on workers' health, such as hypertension, cardiovascular disease, stress, depression, musculoskeletal disorders, chronic infections, diabetes, general health complaints and all-cause mortality in a variety of occupational groups. ${ }^{18-26}$ In physicians, there is some evidence suggesting that LWH can result in impacts such as stress, depression, burnout, injuries, fatigue and sleep deprivation, ${ }^{27-29}$ and overwork has been considered one of the most stressful features of physicians' work. ${ }^{27}$ Some evidence exists of the beneficial effect of reducing the number of working hours on the quality of life of medical residents ${ }^{30}$ and on burnout. ${ }^{27}$

However, the relationship between work and health is complex. There is a substantial body of evidence showing that worklessness is associated with poorer health, whereas work is generally good for health and well-being, ${ }^{31} 32$ provided that the work environment is reasonably acceptable and supportive. ${ }^{33}$

Occupational and non-occupational exposures may play an important role such as the pattern and distribution of working hours, breaks and recovery periods. Also, psychosocial and organisational factors such as workload, job control, managers and peer support, training opportunities, and individual characteristics and attitudes may be important. ${ }^{634}$
Finally, physicians are a highly qualified, devoted and motivated professional group. Despite other existing reviews of a more general occupational approach, a review focused on physicians is warranted.

The aim of this project was to systematically review whether $\mathrm{LWH}$, defined as more than the $48 \mathrm{~h}$ /week limit imposed by the EWTD, are associated with health effects, classified under the International Classification of Diseases (ICD-10) ${ }^{35}$ in physicians, and to examine what these associations are. This review does not include the potential impact of $\mathrm{LWH}$ on patient care or physician training.

\section{METHODS}

The systematic review sought to respond to the following research question: Are $\mathrm{LWH}$ associated with health effects in physicians? For the purpose of this review, exposure to $\mathrm{LWH}$ was defined as working for more than $48 \mathrm{~h} /$ week. $^{1}$ Health outcomes included any disease as defined in the ICD- $10^{35}$ and work-related injuries. If selfassessed tools on health status were used, only those validated were considered. Burnout, stress and outcomes such as individual symptoms, signs or biological markers (blood pressure, ECG, etc) were not considered. Physicians of any medical, surgical or community specialty working in any possible setting (hospitals, primary healthcare, etc), as well as trainees, residents, junior house officers or postgraduate interns, were included.

\section{Study identification}

Electronic searches were carried out using PubMed and EMBASE as search engines (December 2011). Our search strategy was similar in both databases and consisted of a combination of the following keywords and MeSH terms: night shift, morning shift, evening shift, afternoon shift, shift work, rotating shift, shift combination, shift duration or length, shift system, clockwise rotation, shift roster, extended shifts, night work, evening work, work schedule, work hours, starting time, early start, irregular working hours, direction of rotation, overwork, extended hours, shift rota, workload, work schedule tolerance, sleep deprivation, sleep disorders, chronobiology disorders, circadian rhythm, psychomotor performance, circadian disruption, vigilance, alertness, wakefulness, drowsiness, fatigue, insomnia, hypersomnolence, dyssomnia, eveningness, morningness, neurocognitive performance, concentration difficulties, arousal, health, morbidity, mortality, disease, illness, stress, strain, distress, accident, injur*, death, suicid*, education, medical, physician, medical staff, hospital, doctor, surgeon, house officers, medical school, surgery, surgical.

In addition, the reference lists of articles selected for inclusion were carefully reviewed to identify additional studies.

\section{Study selection}

Studies were included if they measured the association between the exposure to LWH and health effects in 
physicians, and used an observational epidemiological (ie, cohort, case-control, cross-sectional) or experimental design. Articles relating to on-call, night or shift work, but with unknown exposure or exposed to less than 48 working hours per week were excluded. They were also excluded if the working groups were other than physicians or addressed other exposures or outcomes (ie, patient safety, fatigue, sleep deprivation, social or family disruption). No limitation was set for languages.

A total of 2036 citations were obtained from PubMed and 980 non-duplicated citations using EMBASE, yielding a total of 3016 citations that were all screened by independent pairs of reviewers. All titles and, where necessary, abstracts were reviewed. Ninety-two potentially suitable publications were identified from the electronic searches, and 18 further studies were identified from the reference lists, yielding a total of 110 studies published in English, French, German, Italian, Slovenian and Spanish. For all of them, the full text was obtained and reviewed by independent pairs of reviewers. Disagreements within pairs were resolved by discussion and, where necessary, by a third reviewer who made the final decision. All authors participated as reviewers for screening the citations and full papers using well-defined and pre-established criteria. Ninety-nine articles were excluded at this stage. One further study was identified that was published after the search period. Finally, 12 papers were considered for quality assessment (figure 1).

The methodological quality of the 12 studies was assessed by independent pairs of reviewers. A standardised 16-point scale based on CONSORT and STROBE statements and adapted from a previous systematic review $^{36}$ was used. It includes 16 items grouped into six areas: (1) objectives; (2) study design; (3) target population and sample; (4) variables; (5) data sources, collection and measurement and (6) statistical methods. Each item was rated as 1 (the requirement was met), 0.5 (the requirement was partially met) or 0 (the requirement was not or unclearly met). Disagreements within pairs of reviewers were resolved by consensus or, where necessary, by a third reviewer. For each of the 12 studies, a final score based on the sum of all items was assigned and the percentage was calculated based on a maximum score of 16 . Study quality was rated as low, moderate or high if it scored less than $60 \%$, between $60 \%$ and $79.9 \%$, and $80 \%$ or more of the maximum score, respectively.

One article of low quality was excluded, ${ }^{37}$ and a total of 11 studies of intermediate or high methodological quality were included for the purpose of this review (figure 1).

\section{Data extraction}

Selected information was extracted from each paper, including publication year, country of origin, study design, setting, study population, sample size, response rate, measure of working hours, definition used for $\mathrm{LWH}$, health outcomes and their measurement, main results on point risk estimates or frequencies of health outcomes, their corresponding 95\% CI and whether the analyses were adjusted for potential confounders.

\section{Evidence synthesis}

To summarise the results on the relationship between LWH and health effects, levels of evidence synthesis was performed. This was based on the methodological quality, study design and consistency of the study outcomes. The following criteria were based on two previous relevant systematic reviews: ${ }^{38} 39$

Strong evidence: consistent results in more than two studies of high quality.

Moderate evidence: consistent results in one highquality study and one intermediate, or between some studies of intermediate quality.

Insufficient evidence: identification of only one study or inconsistent results across studies.
Figure 1 Results of the search strategy, using search engines on PubMed and EMBASE

(December 2011) and screening of references lists of identified full papers, study selection and quality assessment.
3,016 citations obtained from search strategies (2,036 PubMed, 980 Embase)

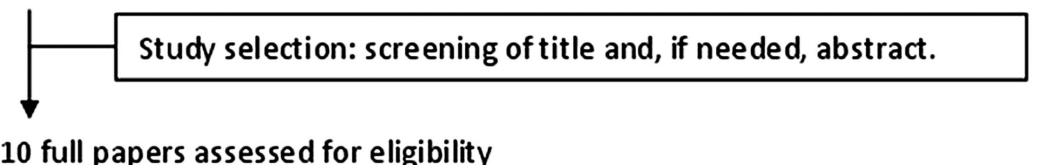

110 full papers assessed for eligibility

( 92 from electronic searches +18 from references lists)

Study selection: inclusion criteria

1 additional study published in 2012 (January) was identified

12 studies selected

Quality assessment.
Exclusion of one study of low quality.

11 studies included in the review 
Evidence of no association: consistent results of a nonassociation in two or more studies.

A measure of the possible magnitude of the association was attempted using the following criteria based on the association point estimate $(\mathrm{RR}=$ relative risk, rate ratio or OR) when available; otherwise the comparison between two frequencies was used:

- High: RR >3.00;

- Intermediate: $\mathrm{RR}=1.50-3.00$;

- Low: $\mathrm{RR}=1.01-1.49$;

- No association: $\mathrm{RR}=1.00$;

- Unclear.

\section{RESULTS}

We identified 11 studies related to $\mathrm{LWH}$ and health effects in physicians that were eligible for inclusion. The outcome of the methodological quality assessment is given in table 1.

Methodological quality was appraised as high for 3 of the 11 studies, with scores ranging from $84 \%$ to $94 \%$. The other eight studies were considered as intermediate quality as their scores ranged from $63 \%$ to $78 \%$. The majority of all included studies received positive scores on items describing the study objectives, design and the study population (items from areas 1, 2 and 3), as well as the description of exposure and outcome variables (item 4). However, although data sources and collection were well described, reliability and validity of exposure to LWH and health outcome measures had low scores (items $5 \mathrm{~b}$ and $5 \mathrm{c}$ ). The statistical methods were in general appropriate; however, the confounding variables were not measured in several studies and, as can be seen, the studies did not describe how they addressed missing data (items $6 \mathrm{a}$ and $6 \mathrm{~b}$ ).

\section{Study characteristics}

The characteristics of the included studies are described in table 2 .

Five had been carried out in North America (four in the USA $^{40} \quad 41 \quad 43 \quad 47$ and one in Canada ${ }^{50}$ ), five in European countries (Denmark, ${ }^{49}$ Germany, ${ }^{45}$ Norway and Germany, ${ }^{43}$ Sweden ${ }^{48}$ and the $\mathrm{UK}^{42}$ ) and one study in Japan. ${ }^{44}$ Four used a prospective cohort design (follow-up ranging from 6 to 20 months), two were casecrossover, four cross-sectional and one was a before and after study. Overall, the total number of participants was 14338 and included medical residents, junior doctors or house officers only working in hospitals, medical specialists or consultants, medical and dental practitioners, general practitioners, hospital physicians and one study included female physicians from a community service. Most studies ( $n=9)$ compared different working hour patterns within the same group of physicians, one compared physicians from two different countries ${ }^{46}$ and one included other healthcare workers as the comparison group. ${ }^{43}$
LWH was defined as more than $48 \mathrm{~h}$ /week in most studies, and some did not provide a definition. Two studies considered LWH below $48 \mathrm{~h} /$ week. $^{46}{ }^{48}$ All the included studies investigated health effects associated with working more than $48 \mathrm{~h} /$ week, with number of hours ranging between $<30$ and $110 \mathrm{~h} /$ week. One paper studied the effects of an increasing number of hours from 30 to more than $50 \mathrm{~h} /$ week $^{44}$ Others studied more than $40,{ }^{49} 47^{48}$ and above $58 \mathrm{~h} /$ week $^{46}$ (two studies), and six studies referred to more than $70 \mathrm{~h} /$ week $^{40-43} 4750$ one of which compared $78-80 \mathrm{~h} /$ week with $90-110$ weekly hours. ${ }^{47}$ Information on working hours was selfreported in nine studies, with reasonably good validity only in two studies ${ }^{40}{ }^{41}$ and reliability in one. ${ }^{43}$ In the other two studies, it was obtained from established work schedules. ${ }^{47} 50$

Identified health outcomes that fulfilled the inclusion criteria were percutaneous injuries, motor vehicle accidents, mood disorders and general health. Information was self-reported in all studies using well-known validated questionnaires, except three studies that used documented information on motor vehicle crashes,${ }^{40}$ reported percutaneous injuries ${ }^{42}$ and antidepressant prescription data as a surrogate of depression. ${ }^{49}$

\section{Summary of findings}

Table 3 shows the findings from each of the 11 studies.

Percutaneous injuries: One study of high quality and one of intermediate quality, with non-adjusted analyses, showed consistent results in medical trainees. Ayas et a $t^{40}$ found an increased risk of percutaneous injuries associated with working more than $20 \mathrm{~h}$ /day compared with non-extended hour periods, except in intensive care units. Fisman et $a l^{43}$ found an association between selfreported fatigue and the number of working hours at the time of injury and a threefold increased risk of sharp injuries in medical trainees compared with other healthcare workers (median of working hours/week before the injury: 70 vs $40 ; p<0.001)$.

Motor vehicle accidents: Two studies of high and intermediate quality showed that long working weeks were associated with an at least twofold increased risk. Barger et $a l^{41}$ found adjusted increased risks for car crashes (OR 2.3; 95\% CI 1.6 to 3.3) and near miss accidents (OR 5.9; $95 \%$ CI 5.4 to 6.3 ) associated with working extended shifts. Kirkcaldy et $a l^{45}$ showed that non-adjusted incidence rates of traffic accidents on house visits was five times as much when physicians worked for a mean of $58 \mathrm{~h} /$ week compared with $38 \mathrm{~h} /$ week $(\mathrm{p}<0.05)$.

Mood disorders: Six studies of intermediate and high quality found contradictory results for mood disorders. Three of them, all of intermediate quality, provided evidence of an association between LWH and mental health problems. The prevalence of General Health Questionnaire (GHQ)-30 cases was higher for female physicians working above $50 \mathrm{~h} /$ week than for those working 30 or less hours/week $(\mathrm{p}<0.05),{ }^{44}$ and an increased adjusted risk of impaired mental health was 


\begin{tabular}{|c|c|c|c|c|c|c|c|c|c|c|c|c|c|}
\hline \multicolumn{3}{|c|}{ Study ID } & \multirow{2}{*}{$\begin{array}{l}\begin{array}{l}\text { Ayas } \\
\text { et }\left.a\right|^{40}\end{array} \\
1\end{array}$} & \multirow{2}{*}{$\begin{array}{l}\text { Barger } \\
\text { et } a^{41}\end{array}$} & \multirow{2}{*}{$\begin{array}{l}\text { Firth- } \\
\text { Cozens }^{42}\end{array}$} & \multirow{2}{*}{$\begin{array}{l}\begin{array}{l}\text { Fisman } \\
\text { et } a f^{43}\end{array} \\
1\end{array}$} & \multirow{2}{*}{$\begin{array}{l}\text { Hayasaka } \\
\text { et } a f^{44} \\
1\end{array}$} & \multirow{2}{*}{$\begin{array}{l}\text { Kirkcaldy } \\
\text { et } a{ }^{45}\end{array}$} & \multirow{2}{*}{$\begin{array}{l}\text { Rosta and } \\
\text { Aasland }^{46}\end{array}$} & \multirow{2}{*}{$\begin{array}{l}\text { Stamp } \\
\text { et } a f^{7}\end{array}$} & \multirow{2}{*}{$\begin{array}{l}\begin{array}{l}\text { Sundquist } \\
\text { and } \\
\text { Johansson }\end{array} \\
1\end{array}$} & \multirow{2}{*}{$\begin{array}{l}\text { Varma } \\
\text { et } a f^{49}\end{array}$} & \multirow{2}{*}{$\begin{array}{l}\begin{array}{l}\text { Zahrai } \\
\text { et } a F^{50}\end{array} \\
1\end{array}$} \\
\hline 1 & Objectives & $\begin{array}{l}\text { Are the objectives or hypotheses of } \\
\text { the research described in the paper? }\end{array}$ & & & & & & & & & & & \\
\hline 2 & Study design & Is the study design presented? & 1 & 1 & 1 & 1 & 1 & 1 & 1 & 1 & 1 & 1 & 1 \\
\hline $3 a$ & $\begin{array}{l}\text { Target } \\
\text { population }\end{array}$ & $\begin{array}{l}\text { Do the authors describe the target } \\
\text { population they wanted to research? }\end{array}$ & 1 & 1 & 1 & 1 & 1 & 1 & 1 & 1 & 1 & 1 & 1 \\
\hline $3 b$ & Sample & $\begin{array}{l}\text { Was a random sample of the target } \\
\text { population taken? AND was the } \\
\text { response rate } 60 \% \text { or more? }\end{array}$ & 0.5 & 0.5 & 1 & 0.5 & 0.5 & 0.5 & 0.5 & 1 & 1 & 1 & 0.5 \\
\hline $3 c$ & Sample & Is participant selection described? & 1 & 1 & 1 & 1 & 0.5 & 1 & 0 & 1 & 1 & 1 & 1 \\
\hline $3 d$ & Sample & $\begin{array}{l}\text { Is participant recruitment described, } \\
\text { or referred to? }\end{array}$ & 1 & 1 & 1 & 1 & 1 & 1 & 1 & 1 & 1 & 1 & 0 \\
\hline $3 e$ & Sample & $\begin{array}{l}\text { Are the inclusion and/or exclusion } \\
\text { criteria stated? }\end{array}$ & 1 & 1 & 1 & 1 & 0.5 & 0.5 & 1 & 1 & 1 & 1 & 0.5 \\
\hline $3 f$ & Sample & $\begin{array}{l}\text { Is the study sample described? } \\
\text { (minimum description: sample size, } \\
\text { gender, age and occupation) }\end{array}$ & 1 & 1 & 0 & 1 & 1 & 1 & 0 & 0 & 0 & 1 & 1 \\
\hline $3 g$ & Sample & $\begin{array}{l}\text { Is the number of participants at each } \\
\text { stage of the study reported? }\end{array}$ & 1 & 1 & 1 & 0.5 & 1 & 0.5 & 1 & 1 & 1 & 1 & 1 \\
\hline 4 & Variables & $\begin{array}{l}\text { Are the measures of long working } \\
\text { hours and the health outcome } \\
\text { described? }\end{array}$ & 1 & 1 & 0.5 & 1 & 1 & 1 & 1 & 1 & 1 & 1 & 1 \\
\hline $5 a$ & $\begin{array}{l}\text { Data sources, } \\
\text { collection }\end{array}$ & $\begin{array}{l}\text { Do authors describe the source of } \\
\text { their data (eg, official registry, health } \\
\text { survey) AND how were the data } \\
\text { collected? }\end{array}$ & 1 & 1 & 1 & 1 & 1 & 1 & 1 & 1 & 1 & 1 & 1 \\
\hline $5 b$ & Measurement & $\begin{array}{l}\text { Was reliability of the measure(s) of } \\
\text { long working hours mentioned or } \\
\text { referred to? }\end{array}$ & 0 & 0 & 0 & 1 & 0 & 0 & 0 & 1 & 0 & 1 & 0 \\
\hline $5 c$ & Measurement & $\begin{array}{l}\text { Was the validity of the measure(s) of } \\
\text { long working hours mentioned or } \\
\text { referred to? }\end{array}$ & 1 & 1 & 0 & 0 & 0 & 0 & 0 & 0 & 0 & 0 & 0 \\
\hline $5 d$ & Measurement & $\begin{array}{l}\text { Were health outcomes assessed by } \\
\text { objective measures or validated } \\
\text { self-reporting instruments? }\end{array}$ & 0 & 1 & 1 & 1 & 1 & 0 & 1 & 1 & 1 & 1 & 1 \\
\hline $6 a$ & $\begin{array}{l}\text { Statistical } \\
\text { methods }\end{array}$ & $\begin{array}{l}\text { Were appropriate statistical methods } \\
\text { used and described, including those } \\
\text { for addressing confounders? }\end{array}$ & 0.5 & 1 & 0.5 & 1 & 0.5 & 0.5 & 1 & 0.5 & 1 & 1 & 0.5 \\
\hline $6 b$ & $\begin{array}{l}\text { Statistical } \\
\text { methods }\end{array}$ & $\begin{array}{l}\text { Were the numbers/percentage of } \\
\text { participants with missing data for } \\
\text { long working hours and the health } \\
\text { outcome indicated AND If more than } \\
20 \% \text { of data in the primary analyses } \\
\text { were missing, were methods used to } \\
\text { address missing data? }\end{array}$ & 0 & 0 & 0 & 0.5 & 0 & 0 & 0 & 0 & 0 & 1 & 0.5 \\
\hline & $\begin{array}{l}\text { al score* }(\%) \\
\text { ality rate† }\end{array}$ & & $\begin{array}{l}12.0(75) \\
\text { interm }\end{array}$ & $\begin{array}{l}13.5(84) \\
\text { high }\end{array}$ & $\begin{array}{l}11.0(69) \\
\text { interm }\end{array}$ & $\begin{array}{l}13.5(84) \\
\text { high }\end{array}$ & $\begin{array}{l}11.0(69) \\
\text { interm }\end{array}$ & $\begin{array}{l}10.0(63) \\
\text { interm }\end{array}$ & $\begin{array}{l}10.5(66) \\
\text { interm }\end{array}$ & $\begin{array}{l}12.5(78) \\
\text { interm }\end{array}$ & $\begin{array}{l}12.0(75) \\
\text { interm }\end{array}$ & $\begin{array}{l}15.0(94) \\
\text { high }\end{array}$ & $\begin{array}{l}11.0(69) \\
\text { interm }\end{array}$ \\
\hline
\end{tabular}




\begin{tabular}{|c|c|c|c|c|c|c|c|}
\hline Study ID & $\begin{array}{l}\text { Country of } \\
\text { study } \\
\text { population }\end{array}$ & Design* & Setting & $\begin{array}{l}\text { Participants and sample } \\
\text { size (response rate) }\end{array}$ & Working hours & Health outcomes & $\begin{array}{l}\text { Quality } \\
\text { score (\%) }\end{array}$ \\
\hline Ayas et $a{ }^{40}$ & USA & $\begin{array}{l}\text { Cohort, prospective } \\
\text { (1 year)/case } \\
\text { cross-over }\end{array}$ & Hospital & $\begin{array}{l}\text { Interns in postgraduate } \\
\text { residency programs } \\
\text { Sample size: } 2737 \text { (80\%) }\end{array}$ & $\begin{array}{l}\text { Mean (SD) hours worked/month: } \\
249.8 \text { (75.3). Self-reported, } \\
\text { monthly survey. Strong } \\
\text { correlation (Pearson } r=0.76 ; p \\
0.001 \text { ) with hours worked } 244 \\
\text { (69.3) from work diaries of } \\
\text { randomly selected subset of } 192 \\
\text { interns. Extended periods ( } 20 \text { or } \\
\text { more consecutive hours) vs } \\
\text { non-extended periods ( } 12 \text { h or } \\
\text { less consecutive hours) }\end{array}$ & $\begin{array}{l}\text { Self-reported } \\
\text { percutaneous injuries }\end{array}$ & $12.0(75)$ \\
\hline Barger et $a f^{41}$ & USA & $\begin{array}{l}\text { Cohort, prospective } \\
\text { (1 year) }\end{array}$ & Hospital & $\begin{array}{l}\text { Residents first postgraduate } \\
\text { year (interns) } \\
\text { Sample size: } 2737 \text { (80\%) }\end{array}$ & $\begin{array}{l}\text { Mean (SD) hours worked/week } \\
70.7(26.0) . \text { Extended shift } \\
\text { ( } \geq 24 \text { h) vs non-extended shift } \\
\text { (<24 h). Self-reported, validated }\end{array}$ & $\begin{array}{l}\text { Self-reported and } \\
\text { documented motor } \\
\text { vehicle crashes }\end{array}$ & $13.5(84)$ \\
\hline Firth-Cozens ${ }^{42}$ & UK & $\begin{array}{l}\text { Cohort, prospective } \\
\text { (1 year) }\end{array}$ & Hospital & $\begin{array}{l}\text { Junior house officers } \\
\text { Sample size: } 170 \text { (72\%) }\end{array}$ & $\begin{array}{l}\text { Mean number of hours/ } \\
\text { week=90.6 } \mathrm{h} \text { (include on call). } \\
\text { Self-reported }\end{array}$ & $\begin{array}{l}\text { GHQ-12 (case: score } \\
\geq 2 \text { ) and SCLDS, } \\
\text { self-reported }\end{array}$ & $11.0(69)$ \\
\hline Fisman et $a{ }^{43}$ & $\begin{array}{l}\text { USA and } \\
\text { Canada }\end{array}$ & Case-crossover & Hospital & $\begin{array}{l}\text { Medical trainees vs other } \\
\text { HCW. } \\
\text { Sample size: } 109 \text { vs } 241 \\
(46 \%)\end{array}$ & $\begin{array}{l}\text { Median number of hours per } \\
\text { week: medical trainees }=70 \text {; } \\
\text { other } \mathrm{HCW}=40(p<0.001) \text {. } \\
\text { Self-reported, high reliability }\end{array}$ & $\begin{array}{l}\text { Reported } \\
\text { percutaneous injuries } \\
\text { to employee } \\
\text { healthcare service }\end{array}$ & $13.5(84)$ \\
\hline $\begin{array}{l}\text { Hayasaka } \\
\text { et } a l^{44}\end{array}$ & Japan & Cross-sectional & $\begin{array}{l}\text { Hospital, } \\
\text { clinics, } \\
\text { other }\end{array}$ & $\begin{array}{l}\text { Female physicians } \\
\text { Sample size: } 367 \text { (63\%) }\end{array}$ & $\begin{array}{l}\text { Comparison of increasing } \\
\text { number of hours/week from } \leq 30 \\
\text { to }>50 \text {, self-reported }\end{array}$ & $\begin{array}{l}\text { GHQ-30 (case: score } \\
\geq 8 \text { ), self-reported }\end{array}$ & $11.0(69)$ \\
\hline Kirkcaldy et a/ ${ }^{45}$ & Germany & Cross-sectional & $\begin{array}{l}\text { Not } \\
\text { specified }\end{array}$ & $\begin{array}{l}\text { Medical and dental } \\
\text { practitioners. Sample size: } \\
2500 \text { (not specified) }\end{array}$ & $\begin{array}{l}\text { Long hours: mean } 58.36 \mathrm{~h} / \text { week } \\
\text { SD } 9.16 \\
\text { Short hours: mean } 38.17 \mathrm{~h} / \text { week } \\
\text { SD } 7.72 \\
\text { Self-reported }\end{array}$ & $\begin{array}{l}\text { Self-reported traffic } \\
\text { accidents }\end{array}$ & $10.0(63)$ \\
\hline $\begin{array}{l}\text { Rosta and } \\
\text { Aasland }^{46}\end{array}$ & $\begin{array}{l}\text { Germany } \\
\text { and Norway }\end{array}$ & Cross-sectional & Hospital & $\begin{array}{l}\text { Hospital physicians } \\
\text { Participants: } 1917(58 \%) \text { in } \\
\text { Germany and } 1072(65 \%) \text { in } \\
\text { Norway answered the } \\
\text { questionnaire. } \\
\text { From survey responders, } \\
1260(65.7 \%) \text { Germans and } \\
562(52.4) \text { Norwegians were } \\
\text { included in the analysis after } \\
\text { applying further inclusion } \\
\text { criteria }\end{array}$ & $\begin{array}{l}\text { German vs Norwegian } \\
\text { physicians }(\%) \text { : } \\
\text { Hours per day }>9=58.8 \text { vs } 26.7 \\
60 \text { h on-call per month }=63.4 \text { vs } \\
18.3 \\
\text { Self-reported }\end{array}$ & $\begin{array}{l}\text { Validated } \\
\text { questionnaire on } \\
\text { self-rated health }\end{array}$ & $10.5(66)$ \\
\hline
\end{tabular}




\begin{tabular}{|c|c|c|c|c|c|c|c|}
\hline Study ID & $\begin{array}{l}\text { Country of } \\
\text { study } \\
\text { population }\end{array}$ & Design* & Setting & $\begin{array}{l}\text { Participants and sample } \\
\text { size (response rate) }\end{array}$ & Working hours & Health outcomes & $\begin{array}{l}\text { Quality } \\
\text { score (\%) }\end{array}$ \\
\hline$\overline{\text { Stamp et } a f^{47}}$ & USA & Before-and-after & Hospital & $\begin{array}{l}\text { Residents of general surgery } \\
\text { Sample size: } 28(97 \%)\end{array}$ & $\begin{array}{l}\text { Changes of work patterns, after } \\
\text { reduction from } 90-110 \text { to } 78-80 \\
\text { work hours/week }\end{array}$ & $\begin{array}{l}\text { SF-36, mental } \\
\text { health; BDI, } \\
\text { depression; } \\
\text { self-reported }\end{array}$ & $12.5(78)$ \\
\hline $\begin{array}{l}\text { Sundquist and } \\
\text { Johansson } 48\end{array}$ & Sweden & Cross-sectional & $\begin{array}{l}\text { Primary } \\
\text { care }\end{array}$ & $\begin{array}{l}\text { General practitioners } \\
\text { Sample size: } 1004(72 \%)\end{array}$ & $\begin{array}{l}\text { Overtime defined as working at } \\
\text { least } 47 \mathrm{~h} / \text { week. Self-reported }\end{array}$ & $\begin{array}{l}\text { Swedish SF-36, } \\
\text { impaired mental } \\
\text { health, self-reported }\end{array}$ & $12.0(75)$ \\
\hline Varma et $a f^{49}$ & Denmark & $\begin{array}{l}\text { Cohort, prospective } \\
\text { (20 months) }\end{array}$ & Hospital & $\begin{array}{l}\text { Senior medical consultants } \\
\text { Sample size: } 2790(62 \%)\end{array}$ & $\begin{array}{l}\text { Long work hours (>40 h/week). } \\
\text { Self-reported }\end{array}$ & $\begin{array}{l}\text { Depression: } \\
\text { redemption of } \\
\text { anti-depressive drug } \\
\text { prescriptions }\end{array}$ & $15.0(94)$ \\
\hline Zahrai et $a^{50}$ & Canada & $\begin{array}{l}\text { Cohort, prospective } \\
\text { (6 months) }\end{array}$ & Hospital & $\begin{array}{l}\text { Orthopaedic surgery } \\
\text { residents } \\
\text { Sample size: } 16 \text { (not } \\
\text { specified) }\end{array}$ & $\begin{array}{l}\text { Night float }(n=9): 77.8 \% \text { did } \\
>80 \text { h/week at baseline; } 71.4 \% \\
\text { at follow-up } \\
\text { Standard call }(n=7): 57.1 \% \text { did } \\
>80 \text { h/week at baseline; } 80 \% \text { at } \\
\text { follow-up }\end{array}$ & $\begin{array}{l}\text { SF-36, mental health } \\
\text { score, self-reported }\end{array}$ & $11.0(69)$ \\
\hline
\end{tabular}

${ }^{*}$ Follow-up period in brackets for prospective cohort studies.

BDI, Beck Depression Inventory II; GHQ-12, General Health Questionnaire-12 items; HCW, Health Care Workers; SCLDS, Symptom Checklist Depression Scale. 
Table 3 Results on the association between long working hours and identified health effects in physicians

\begin{tabular}{|c|c|c|c|}
\hline Health outcome & Main results & Adjustment by confounders & Study ID \\
\hline Percutaneous injuries & $\begin{array}{l}\text { OR }(95 \% \mathrm{Cl}) \text { for injuries during extended ( }>20 \mathrm{~h} / \text { day) vs non-extended periods (first } \\
12 \mathrm{~h} \text { at work): all percutaneous injuries: } 1.61(1.46 \text { to } 1.78) \text {; injuries reported to } \mathrm{OH} \text { : } \\
1.83(1.48 \text { to } 2.28) \text {; injuries in the ICU: } 1.87 \text { (0.69 to } 5.04) \text {; injuries in the operating } \\
\text { room or labour and delivery: } 1.77 \text { ( } 1.49 \text { to } 2.09) \text {; injuries in the ICU, non-ICU or ED: } \\
2.17(1.56 \text { to } 3.00)\end{array}$ & Time of day and circadian influences & Ayas et $a f^{40}$ \\
\hline Percutaneous injuries & $\begin{array}{l}\text { Medical trainees vs other healthcare workers: total median working hours: } 70 \text { vs } \\
40 \mathrm{~h}(\mathrm{p}<0.001) \text {; median previous working hours at the time of injury: } 6.5 \mathrm{vs} 5 \mathrm{~h} \\
(\mathrm{p}<0.001) \text {. } \\
\text { OR }(95 \% \mathrm{Cl}) \text { for self-reported fatigue: work }>40 \mathrm{~h} \text { in the past week: } 3.50 \text { ( } 2.06 \text { to } \\
5.92) ; \text { work during more than } 5 \mathrm{~d} \text { in past week: } 4.20 \text { ( } 4.46 \text { to } 7.15) ;>12 \mathrm{~h} \text { at work } \\
\text { before injury: } 8.58(3.7 \text { to } 19.86) \text {. } \\
\text { Incidence rate ratio }(95 \% \mathrm{Cl}) \text { for association between fatigue and injuries: all } \\
\text { workers: } 1.40(1.03 \text { to } 1.90) \text {; medical trainees: } 2.94(1.71 \text { to } 5.07) \text {; other HCWs: } 0.97 \\
\text { (0.66 to } 1.42 ; p=0.001 \text { for heterogeneity) }\end{array}$ & Age and sex & Fisman et $a f^{43}$ \\
\hline Motor vehicle accidents & $\begin{array}{l}\text { ORs }(95 \% \mathrm{Cl}) \text {, after extended shift }(\geq 24 \mathrm{~h}) \text { vs non-extended shift }(<24 \mathrm{~h}) \text { : } \\
\text { Crashes }=2.3(1.6 \text { to } 3.3) ; \text { near miss accidents }=5.9(5.4 \text { to } 6.3)\end{array}$ & Age and sex & Barger et $a f^{41}$ \\
\hline Motor vehicle accidents & $\begin{array}{l}\text { Incidence rates of driving accidents on house visits: } \\
\text { Long hours (mean } 58.36 \mathrm{~h} / \text { week)=0.10 }(0.31) \text { vs short hours (mean } 38.17 \mathrm{~h} / \text { week) } \\
=0.02(0.18) . \mathrm{p}<0.05\end{array}$ & Not adjusted & Kirkcaldy et $a f^{45}$ \\
\hline $\begin{array}{l}\text { Mood disorders, } \\
\text { depression }\end{array}$ & $\begin{array}{l}\mathrm{HR}(95 \% \mathrm{Cl}) \text { hours/week intervals and redemption of antidepressive drug } \\
\text { prescription(reference group: } 37-40 \mathrm{~h} / \text { week): } \\
25-36 \mathrm{~h}: 0.83(0.24 \text { to } 2.82) ; 41-44 \mathrm{~h}: 0.95(0.50 \text { to } 1.77) ; 45-49 \mathrm{~h}: 0.88(0.43 \text { to } \\
1.78) ; 50-54 \mathrm{~h}: 0.83 \text { (0.32 to } 2.14) ; 55-59 \mathrm{~h}: 0.67(0.15 \text { to } 2.94) ;>60 \mathrm{~h}: 0.48 \\
(0.06 \text { to } 3.68) \text {. } \\
\text { Cox regression analysis of work hours as a continuous variable: } \mathrm{HR}=0.93 \\
\text { (0.76 to } 1.13 \text { ) }\end{array}$ & $\begin{array}{l}\text { Age, sex, marital status, medical specialty, } \\
\text { decision authority at work, social support at } \\
\text { work, quantitative work demands and } \\
\text { previous redemption of } A D \text { drug prescription }\end{array}$ & Varma et $a f^{49}$ \\
\hline $\begin{array}{l}\text { Mood disorders, } \\
\text { GHQ-30 }\end{array}$ & $\begin{array}{l}\text { Prevalence of cases by working time (hours/week) } \\
\leq 30 \text { h: } 35.7 \%(41 / 115) ;>30-40 \text { h: } 39.0 \%(39 / 100) ;>40-50 \text { h: } 37.7 \%(26 / 69) ;>50 \text { h: } \\
56.8 \%(46 / 81) ; p=0.0179 \\
\text { Stepwise multiple logistic regression, }>50 \text { vs } \leq 50 \text { h/week: parameter } 0.635 \\
\text { (p=0.0293) }\end{array}$ & $\begin{array}{l}\text { Marital status, medical facility, position and } \\
\text { night duty }\end{array}$ & Hayasaka et a ${ }^{44}$ \\
\hline $\begin{array}{l}\text { Mood disorders, } \\
\text { GHQ-12 and SCLDS }\end{array}$ & $\begin{array}{l}\text { No association was found between number of hours worked in a week and } \\
\text { depression }\end{array}$ & Not adjusted & Firth-Cozens ${ }^{42}$ \\
\hline Mood disorders, SF-36 & $\begin{array}{l}\text { Scores when night float vs standard call at baseline and follow-up: } \\
\text { Mental health mean score (SD): baseline= }=57.33(22.63) \text { vs } 65.71(7.61) \text {; } \\
\text { follow-up=52.00 (15.49) vs } 60.80(11.45) ; p=0.72 \\
\text { Mental health component summary (SD), baseline=34.84 (14.06) vs } 40.21 \text { (7.61); } \\
\text { follow-up=30.15 (10.71) vs } 42.40(6.23) ; p=0.39 \\
\text { Regression analysis: increased number of hours in hospital correlated with } \\
\text { significantly lower SF-36 scores in almost all domains }\end{array}$ & Not adjusted & Zahrai et $a^{50}$ \\
\hline
\end{tabular}




\begin{tabular}{|c|c|c|c|}
\hline Health outcome & Main results & Adjustment by confounders & Study ID \\
\hline $\begin{array}{l}\text { Mood disorders, SF-36 } \\
\text { and BDI }\end{array}$ & $\begin{array}{l}\text { Improvement of levels before and after duty hours limitations from } 90-110 \text { to } 78- \\
80 \mathrm{~h} / \text { week): } \\
\text { Mental health SF-36: no statistically significant improvement } \\
\text { Individual questions BDI before and after duty hours limitations, only energy level } \\
\text { statistically significant }\end{array}$ & Not adjusted & Stamp et $a \uparrow^{47}$ \\
\hline Mood disorders, SF-36 & $\begin{array}{l}\text { OR ( } 95 \% \text { Cl)for working } 47 \mathrm{~h} / \text { week or more: } \\
\text { Impaired mental health, men: } 1.59 \text { (0.95 to } 2.66) \text {; women: } 1.86 \text { (1.03 to } 3.37)\end{array}$ & Age and amount of time in practice & $\begin{array}{l}\text { Sundquist and } \\
\text { Johansson } 48\end{array}$ \\
\hline General health, SF-36 & $\begin{array}{l}\text { SF-36: } \\
\text { Scores when night float vs standard call at baseline and follow-up: } \\
\text { General health mean score (SD): baseline=62.11 (17.47) vs } 77.57(24.25) \text {; } \\
\text { follow-up=56.43 (24.89) vs } 84.20(16.50) ; p=0.41 \\
\text { Physical health component summary (SD): baseline=46.16 (13.15) vs } 52.01 \text { (13.33); } \\
\text { follow-up=39.32 }(9.80) \text { vs } 56.15(2.18) ; p=0.015 \\
\text { Vitality mean score (SD): baseline=51.67 (14.58) vs } 51.43 \text { (15.74); follow-up=48.57 } \\
\text { (14.92) vs } 51.00(10.84) ; p=0.20 \\
\text { Regression analysis demonstrated that the increased number of hours spent in } \\
\text { hospital correlated with significantly lower general health, physical function, mental } \\
\text { health, role emotional, social function and mental component summary scale scores } \\
\text { (all } p<0.05)\end{array}$ & Not adjusted & Zahrai et $a^{50}$ \\
\hline General health, SF-36 & $\begin{array}{l}\text { SF-36, BDI: } \\
\text { Improvement of levels before and after duty hours limitations from } 90-110 \text { to } \\
78-80 \mathrm{~h} / \text { week: } \\
\text { Physical health: no statistically significant differences }\end{array}$ & Not adjusted & Stamp et $a{ }^{47}$ \\
\hline General health, SF-36 & $\begin{array}{l}\text { SF-36: } \\
\text { OR }(95 \% \mathrm{Cl}) \text { adjusted for age and time in practice, for working } 47 \mathrm{~h} / \text { week or more: } \\
\text { Impaired general health: men=1.66 (1.00 to } 2.77) \text {; women }=1.59(1.00 \text { to } 3.17)\end{array}$ & Age and amount of time in practice & $\begin{array}{l}\text { Sundquist and } \\
\text { Johansson } 48\end{array}$ \\
\hline General health & $\begin{array}{l}\text { Self-rated health: } \\
\text { OR }(95 \% \mathrm{Cl}) \text { of good self-rated health (logistic regression): } \\
\text { Norwegian work time pattern }{ }^{\star}=1.35(1.03 \text { to } 1.77) \text {; working in Norway }=4.17 \\
(3.02 \text { to } 5.73)\end{array}$ & Age, sex and country of work & $\begin{array}{l}\text { Rosta and } \\
\text { Aasland } 46\end{array}$ \\
\hline
\end{tabular}

${ }^{*}$ Not working more than $9 \mathrm{~h}$ a day and having more than $60 \mathrm{~h}$ a month on-call.

AD, anti-depressive (Mood disorders, depression); BDI, Beck Depression Inventory II; ED, Emergency Department; GHQ-12, General Health Questionnaire-12 items; ICU, intensive care unit; $\mathrm{OH}$, Occupational Health (Percutaneous Injuries); SCLDS, Symptom Checklist Depression Scale. 
found for male and female general practitioners who worked for $47 \mathrm{~h} /$ week or more. $^{48}$ Zahrai $e t a \bar{l}^{0}$ found that increased number of hours spent by residents in hospital correlated significantly with lower mental health and Mental Component Summary Scale scores (SF-36). Three other studies, one of which is of high quality, did not find an association between depression or mood disorders and LWH. Varma et $a t^{49}$ did not find higher adjusted risks of depression associated with increased number of hours in senior medical consultants, even when they worked for more than $60 \mathrm{~h} /$ week. Depression or mood disorders measured with GHQ-12, Symptom Checklist Depression Scale (SCLDS) or Beck Depression Inventory (BDI), all validated tools for mental health, were not associated with increased number of working hours ${ }^{42}$ nor improved after decreasing the number of hours from $90-110$ to $78-80 \mathrm{~h} /$ week in residents. ${ }^{47}$

General health: Four studies of intermediate quality analysed the association between LWH and general health. Zahrai et $a b^{50}$ found that increased number of hours spent by residents in hospital correlated significantly with poorer general health, physical function and vitality using SF-36. A comparative study of physicians in two different countries found that Norwegians showed higher non-adjusted prevalence of self-reported good health compared with physicians in Germany who worked longer hours. ${ }^{46}$ An increased adjusted risk of impaired general health was found for male and female general practitioners who worked for more than $47 \mathrm{~h} /$ week. $^{48}$ However, following the implementation of reducing the number of hours from an average of 90-110 to 78-80 h/ week did not lead to an overall improvement of residents' self-reported physical health. ${ }^{47}$

\section{Synthesis of the evidence}

The levels of evidence synthesis obtained from the analysis of the 11 studies included in this review are shown in table 4 .
There is moderate evidence of an association between LWH and percutaneous and motor vehicle accidents in physicians. This evidence comes from one study of high quality and another of intermediate quality for percutaneous injuries and for motor vehicle accidents; the magnitude of this association could be intermediate.

For mood disorders and general health, there is a low or insufficient level of evidence of an association with LWH in physicians. This comes from six studies on mood disorders and four on general health of high and intermediate methodological quality and inconsistent results among them. No conclusion can be drawn about the magnitude of such associations, even if they existed.

\section{DISCUSSION}

This review found moderate scientific evidence for a positive association of intermediate magnitude between exposure to LWH and percutaneous injuries and motor vehicle accidents in physicians. Evidence was assessed as low or insufficient for mood disorders and general health.

To the best of our knowledge, this is the first systematic review on the effects of LWH on physicians' health and safety. The extensive searches were restricted to indexed journals. We systematically identified, selected and assessed the methodological quality of studies by means of independent pairs of reviewers. The quality assessment form and the levels of evidence used in this review were based on CONSORT and STROBE statements and on previous systematic reviews. ${ }^{36} 3839$ To give some estimate of the potential magnitude of the effect, we added levels based on the point estimates of measures of association provided by the studies. However, such levels had not been previously established.

\section{Strengths and limitations of the studies}

Four studies had a longitudinal prospective design, however only two of them used a robust methodology.

Table 4 Available scientific evidence on the health problems associated with long working hours in physicians: levels of evidence synthesis

\begin{tabular}{|c|c|c|c|}
\hline Health outcome & $\begin{array}{l}\text { Degree of } \\
\text { evidence* }^{*}\end{array}$ & $\begin{array}{l}\text { Magnitude of the } \\
\text { association } \dagger\end{array}$ & Studies ID \\
\hline $\begin{array}{l}\text { Percutaneous } \\
\text { injuries }\end{array}$ & ++ & ++ & Ayas et $a l^{40}$ Fisman et $a f^{43}$ \\
\hline $\begin{array}{l}\text { Motor vehicle } \\
\text { accidents }\end{array}$ & ++ & ++ & Barger et al, ${ }^{41}$ Kirkcaldy et al ${ }^{45}$ \\
\hline Mood disorders & + & \pm & $\begin{array}{l}\text { Varma et } a l,{ }^{49} \text { Hayasaka et al, }{ }^{44} \text { Firth-Cozens, }{ }^{42} \\
\text { Zahrai et } a l,{ }^{50} \text { Stamp et } a l,{ }^{47} \text { Sundquist and Johansson }{ }^{48}\end{array}$ \\
\hline General health & + & \pm & $\begin{array}{l}\text { Zahrai et } a l,{ }^{50} \text { Stamp et al, }{ }^{47} \text { Sundquist and Johansson, }{ }^{48} \\
\text { Rosta and Aasland }\end{array}$ \\
\hline \multicolumn{4}{|c|}{$\begin{array}{l}\text { *Strong evidence (+++): consistent results in more than two studies of high quality; moderate evidence (++): consistent results in two studies } \\
\text { of high quality, or one high-quality study and one intermediate, or between some studies of intermediate quality; insufficient evidence (+): } \\
\text { identification of only one study or inconsistent results across studies; evidence of no association }(-) \text { : consistent results of a non-association in } \\
\text { two or more studies. } \\
+ \text { +Magnitude of the association: it refers to the magnitude of the association point estimate (RR=relative risk, rate ratio or OR): high (+++) if RP } \\
>3 \text {; intermediate (++) if RR=1.5-3; low (+) if RR }<1.5 \text {; no association }(-) \text {; unclear }( \pm) \text {. }\end{array}$} \\
\hline
\end{tabular}


Two other studies used a crossover design, which reduces the likelihood of individual variability and confounding, and were considered well designed. Four studies, all rated as intermediate methodological quality, were cross-sectional in design and therefore it is difficult to draw causal relationships from them. Although reverse causality cannot be ruled out from crosssectional designs, it is unlikely that poorer health determines longer working hours than their healthier peers. One study used a quasi-experimental design but lacked a control group. Also, because of different designs used across the included studies, different estimates of the effects were used that may not be directly comparable.

Other limitations include the variability, validity and reliability of working hours, which in most studies was self-reported. Although only those papers specifying the number of working hours were accepted, shift and night work might have worked as confounders too, as it is difficult to acknowledge which proportion of the health effects observed was due exclusively to the LWH component and not due, for instance, to stress, fatigue or sleep deprivation. Moreover, the studies did not discriminate between time spent actively working and asleep while on call, though the EWTD considers all hours on call as working time. Likewise, it is possible that participants were aware that the study was conducted to assess associations between the number of hours worked and health outcomes. Therefore, there might be a volunteer bias in those doctors who work extended hours. To minimise this bias, a case-crossover analysis has been performed in three of the included studies. ${ }^{40} 4143$

Health outcomes were measured mainly based on selfreport, with the exception of documented motor vehicle crashes, ${ }^{40}$ reported percutaneous injuries ${ }^{42}$ and registernotified antidepressive drug prescriptions. ${ }^{48}$ No studies with other objective measures of health (eg, mortality) or mental disease (eg, hospital data) were identified. However, self-reported or perceived health was assessed by validated and widely used instruments, especially when health status (general, mental or physical health) or ill-health symptoms were evaluated. Nonetheless, this raises the possibility that the observed associations might reflect differences in propensity to report health problems when they occur rather than true differences in the risk of worse health status. We cannot rule this out, but the higher propensity for reporting among those who work for longer hours and poorer work schedule patterns would have to relate specifically to the reporting of ill health rather than non-ill health. It seems unlikely that major differences in propensity to report would extend to a more concrete outcome.

The majority of the studies of this review took into account confounding variables in their analyses, such as sex and age. However, none of the studies analysed the potential effect of modifying factors, such as psychosocial aspects at work, including attitudes, motivation, job requirements, demands and content, organisational climate, social relationships at work, work satisfaction, supportive organisation or the relative number of physicians and other staff available for patient care. Neither were important aspects outside of work, such as life events or lifestyles, taken into account. The study of Japanese female physicians found significant differences by marital status with higher GHQ scores for those who were married. ${ }^{44}$ There are significant differences in the number of physicians per capita across different countries and this together with the structure and organisation of health services are potential confounders too that were not addressed in the selected studies across countries.

A relevant outcome from this review would be to establish a threshold number of extended hours above which there is a significantly higher risk. There is a lack of evidence for a dose-response relationship and nor does the evidence give any indication for a threshold number of hours that physicians could work and remain safe and healthy.

Finally, the heterogeneity of the included studies did not allow a more quantitative synthesis, and a level of evidence approach was used instead.

\section{Comparison with other studies}

Our findings of an association between LWH and injuries and accidents in physicians are consistent with research in other occupational groups. Dembe et al ${ }^{19}$ carried out a longitudinal survey in the USA including more than 10000 workers from a variety of occupations and settings. A strong dose-response effect (adjusted for age, gender, occupation, industry and region) was found between LWH and injuries above $40 \mathrm{~h} /$ week and $8 \mathrm{~h}$ / day; jobs with overtime schedules were associated with a $61 \%$ higher injury hazard rate compared with jobs without overtime; working at least $12 \mathrm{~h}$ /day was associated with a $37 \%$ increased hazard rate, and working at least $60 \mathrm{~h} /$ week was associated with a $23 \%$ increased hazard rate. They concluded that LWH might indirectly precipitate workplace injuries through a causal process by inducing fatigue or stress in workers. In a separate study assessing injury risks to healthcare personnel, Dembe et $a \bar{l}^{1}$ demonstrated that the risk of injury when working overtime or at least $60 \mathrm{~h} /$ week among physicians and nurses was statistically significant. In our review, we found that information about the length of shift work varied across studies, some analysing shifts of up to $24 \mathrm{~h}$. Work that included such shifts was more strongly associated with accidents and injuries. ${ }^{40} 41$

We found insufficient evidence of an association between LWH and mood disorders, other diseases and general health in physicians, despite some scientific evidence suggesting that $\mathrm{LWH}$ increase morbidity and mortality in other occupations. ${ }^{19-26}$

Possible explanations for these inconsistencies might be the scarcity of high-quality research, and the fact that some of the included papers did not fully address the effects of confounding factors, and the possibility that working as a physician might have a protective effect by 
itself, for instance, through higher motivation and social recognition relative to other occupations.

Stamp et $a l,{ }^{47}$ in the only before and after study, did not find significant differences for mood disorder or general health before and after implementing requirements to reduce working schedules to $80 \mathrm{~h}$ /week in residents. It is debatable, however, whether any conclusions can be drawn from the impact of reducing extremely high working hours $(90-110 \mathrm{~h} /$ week) to very high working hours (78-80 h/week).

Although we searched for and identified literature describing associations between LWH and increased mortality in doctors, ${ }^{52} 53$ none of those studies specified the number of hours worked, and therefore were not included.

\section{Recommendations for future research}

Further research, including well-designed prospective and retrospective longitudinal studies, is needed in this field to establish on scientific grounds what number of hours are safe, to patients as well as to physicians. Also, studies on long-term health effects should be considered.

This review was aimed at studying exclusively possible detrimental health effects of LWH (more than $48 \mathrm{~h}$ /week) on physicians. Other systematic reviews should be conducted addressing issues such as the impact of working hours on the well-being and quality of life of doctors, the training quality of junior doctors, and the quality and continuity of care and their impact on patient safety. Also, from a staffing perspective, the economic and social burden of sick leave and physicians leaving the profession are important issues that were beyond the scope of this review and that should be considered in future research. Other established criteria, ${ }^{54}$ such as that working hours should be 'family friendly', promote gender equality, enhance productivity or facilitate worker choice and influence over working hours, were not addressed. Further research is needed in these other areas.

\section{Conclusions}

The findings of this review have confirmed that LWH are associated with an increased risk of needlestick injuries and road traffic accidents. The fact that these associations are labelled moderate in this review should not preclude safety procedures and measures being assessed and reassessed to ensure maximum physician and patient safety. The reduction of needlestick injuries requires improved use of safe sharp devices, compliance with safe working procedures, training and regular audits of working practices, particularly for junior doctors who are at greatest risk of needlestick injuries, possibly due to inadequate training. ${ }^{55} 56$ The risk of road traffic accidents could be reduced by discouraging driving after very long shifts (more than $16 \mathrm{~h}$ ) and where there is sleep deprivation. ${ }^{57}$ Transport should be provided by the employer in these situations. This is a pragmatic recommendation which recognises that LWH have not been eliminated as a result of the EWTD.
This review uncovered the lack of literature on the effect of LWH on the general health of doctors, a topic that has important health ramifications not only for physicians but for patients as well. However, the paucity of available studies, which does not allow for a causal or dose-response relationship to be established, raises further questions about the evidence base of the current $48 \mathrm{~h}$ limit, especially as this is not enforced strictly and junior doctors, for example, in the UK, may opt-out. ${ }^{58} \mathrm{It}$ may be relevant that the EWTD was not based entirely on science but also took into account, as does all EU legislation, the views of 'social partners'?

It is likely that the mechanism for these increased health and safety risks is fatigue, and our finding of increased risk of accidents to staff may also be associated with increased risk of clinical errors. Patient safety was not the purpose of this review but LWH are well recognised to cause decrement in performance in healthcare and other professions, where the performance of staff can be safety critical, such as being airline pilots or professional drivers. ${ }^{59}$

In addition, the EWTD has been associated with real concerns about the adequacy of the training of doctors given their reduced exposure to patient care, and the lack of experience of the patient journey, imposed by adherence to the EWTD. ${ }^{4}$ This is potentially exacerbated at the same time by the constraints on the overall duration of training imposed by other EU directives. ${ }^{60}$ Further reviews of the EWTD, with regard to this unique group of workers, need to take into account other social factors such as the impact on patient care. This is particularly the case when all healthcare systems are under strain because of burgeoning demands and limited resources. $^{4}$

The findings of this systematic review lead to the suggestion of the following guidelines in the event of noncompliance with the EWTD: (1) long shifts should be avoided to protect physicians' health and patient safety and no shift should be longer than $16 \mathrm{~h}$ (and then exceptionally ${ }^{28}$; (2) physicians should be discouraged from driving after long shifts to reduce the risk of motor accidents ${ }^{41}{ }^{45}$; (3) rigorous attention should be paid to reduce the risk of sharp injuries ${ }^{40} 43$; (4) organisational aspects such as workload and job control, as well as the pattern and distribution of working hours, breaks and recovery periods should be carefully taken into account, in order to avoid fatigue and sleep deprivation that could lead to mistakes and accidents ${ }^{61} 62$ and (5) physicians should work in supportive psychosocial environments, in teams and with adequate training and supervision as their well-being is important also for the health of their patients. ${ }^{61} 62$

Further research is required: to establish any longer term effects, such as on mortality and mental health; to assess the impact of the nature of the work organisation taking into account the psychosocial aspects of the physicians' working and non-working lives and to determine how many hours are safe for physicians to work. 
Author affiliations

${ }^{1}$ Catalan and Spanish Societies of Occupational Medicine, Barcelona, Spain ${ }^{2}$ Department of Medical Sciences, School of Medicine, Universitat de Girona, Girona, Spain

${ }^{3} \mathrm{MRC} / \mathrm{CSO}$ Social and Public Health Sciences Unit, University of Glasgow, Glasgow, UK

${ }^{4}$ Healthy Working Lives Group, Institute of Health and Wellbeing, College of Medical, Veterinary and Life Sciences, University of Glasgow, Glasgow, UK ${ }^{5} \mathrm{CiSAL}$ - Centre for Research in Occupational Health, Universitat Pompeu Fabra, Barcelona, Spain

${ }^{6}$ Occupational Health Department, Epsom \& St Helier University Hospitals NHS Trust, Surrey, UK

${ }^{7}$ Clinical Institute of Occupational, Traffic and Sports Medicine, University Medical Centre, Ljubljana, Slovenia

${ }^{8}$ College of Occupational Medicine, Portuguese Medical Association, Lisbon, Portugal

${ }^{9}$ Helsinki City Occupational Health Centre, Helsinki, Finland

${ }^{10}$ Occupational Health Service, Parc de Salut MAR, Barcelona, Spain

${ }^{11} \mathrm{CIBER}$ of Epidemiology and Public Health, Spain

${ }^{12}$ UEMS Section of Occupational Medicine

Acknowledgements Leena Isotalo, Trial Search Coordinator at the Cochrane Occupational Safety and Health Review Group, designed and conducted the final search of the evidence using MEDLINE and EMBASE databases.

Contributors MCR-J, ED, SV-P, AŠ, KAS, EBM and CS conceived and submitted the design, carried out the acquisition of data, analysis and interpretation of data, drafted the article and revised it. PGR and R H-A contributed to acquisition of data, analysis and interpretation of data, drafted the article and revised it. All authors gave final approval of the version to be published, and agree to be accountable for all aspects of the work.

Funding The Section of Occupational Medicine of the UEMS provided limited support to the work of MCR-J, ED, SV-P, KAS, AS, PGR and RH-A. The expenses of one meeting of the core group (MCR-J, SV-P, ED, EBM and CS) were also funded by the Section. Role of funding source: The Section of Occupational Medicine of the UEMS acted in its role of promoting and contributing to the research in Occupational Medicine.

Competing interests None.

Ethics approval

Provenance and peer review Not commissioned; externally peer reviewed.

Data sharing statement No additional data are available.

Open Access This is an Open Access article distributed in accordance with the Creative Commons Attribution Non Commercial (CC BY-NC 3.0) license, which permits others to distribute, remix, adapt, build upon this work noncommercially, and license their derivative works on different terms, provided the original work is properly cited and the use is non-commercial. See: http:// creativecommons.org/licenses/by-nc/3.0/

\section{REFERENCES}

1. EUR-Lex. Directive 2003/88/EC of the European Parliament and of the Council of 4 November 2003 concerning certain aspects of the organisation of working time. In: Union OJotE, ed. Brussels: Official Journal of the European Union, 2003.

2. EPHA. EPHA Briefing on the European Working Time Directive (EWTD). 2012. http://www.epha.org//MG/pdf/EPHA Briefing EWTD_Oct2012_FINAL.pdf (accessed 2012).

3. European Commission. Working Conditions-Working Time Directive. 2013. http://ec.europa.eu/social/main.jsp?catld=706\&langld=en\& intPageld $=205$ (accessed Dec 2013).

4. House J. Calling time on doctors' working hours. Lancet 2009;373:2011-12

5. The Lancet. Doctors' training and the European Working Time Directive. Lancet 2010;375:2121.

6. Leff D, Aziz O, Darzi A. Trucks, planes, and scalpels-is there an evidence-based approach to surgeons' working hours? Arch Surg 2007:142:817-20

7. Parthasarathy S. Sleep and the medical profession. Curr Opin Pulm Med 2005;11:507-12.
8. Dr. Foster Hospital Guide. 2011. http://www.drfosterhealth.co.uk (accessed Feb 2011)

9. UEMS. The Union of European Medical Specialists. 2012. http:// www.uems.net (acessed 2012)

10. Macdonald EB, Ritchie KA, Murray KJ, et al. Requirements for occupational medicine training in Europe: a Delphi study. Occup Environ Med 2000;57:98-105.

11. Macdonald E, Baransky B, Wilford J editors. Occupational Medicine in Europe: Scope and Competencies [Internet]. Bilthoven: WHO European Centre for Environment and Health; 2000. http://www. who.int/occupational_health/regions/en/oeheuroccmedicine.pdf (accessed 6 Feb 2011).

12. Ballester M, Cordell N, Rodriguez Jareno MC, et al. A European survey of professional bodies representing occupational medicine specialists. Occup Med (Lond) 2012;62:366-70.

13. Cashman C, Slovak A. The occupational medicine agenda: routes and standards of specialization in occupational medicine in Europe. Occup Med (Lond) 2005;55:308-11.

14. De Schryver A, Claesen B, Meheus A, et al. European survey of hepatitis B vaccination policies for healthcare workers. Eur J Public Health 2011;21:338-43.

15. Rodriguez-Juareno M, Serra C, Demou E, et al. European Working Time Directive: systematic review for evidence based decision making. Summary Report. In: Medicine USoO, ed. UEMS Section of Occupational Medicine, 2011. http://www.uems-occupationalmedicine. org/sites/defaultffiles/old_userfiles/File\%203\%20-\%20EWTD\% 20UEMS\%20REPORT\%2005\%2010\%202011.pdf

16. UEMS. The Newsletter of European Medical Specialists. 2010. http://www.uems.eu/_data/assets/pdf_file/0014/1463/1466.pdf

17. UEMS. Meetings of the Board \& Council of UEMS- Report. In: European Union of Medical Specialists. http://www.uems.eu/ data/ assets/pdf_file/0011/1235/UEMS_2011.37___report_-_UEMS_ Council 6-8.10.2010 Napoli.pdf, ed. Napoli (Italy), 2011.

18. Bannai $\bar{A}$, Tamakoshi $A$. The association between long working hours and health: a systematic review of epidemiological evidence. Scand J Work Environ Health 2013;40:5-18.

19. Dembe AE, Erickson JB, Delbos RG, et al. The impact of overtime and long work hours on occupational injuries and illnesses: new evidence from the United States. Occup Environ Med 2005;62:588-97.

20. Johnson JV, Lipscomb J. Long working hours, occupational health and the changing nature of work organization. $\mathrm{Am} J$ Ind Med 2006;49:921-9.

21. Kivimaeki M, Batty GD, Hamer M, et al. Using additional information on working hours to predict coronary heart disease. Ann Intern Med 2011;154:457-63.

22. Liu Y, Tanaka H; Fukuoka Heart Study G. Overtime work, insufficient sleep, and risk of non-fatal acute myocardial infarction in Japanese men. Occup Environ Med 2002;59:447-51.

23. Sokejima S, Kagamimori S. Working hours as a risk factor for acute myocardial infarction in Japan: case-control study. BMJ 1998; $317: 775-80$

24. Spurgeon A, Harrington JM, Cooper CL. Health and safety problems associated with long working hours: a review of the current position. Occup Environ Med 1997:54:367-75.

25. Virtanen M, Ferrie JE, Singh-Manoux A, et al. Long working hours and symptoms of anxiety and depression: a 5-year follow-up of the Whitehall II study. Psychol Med 2011;41:2485-94.

26. Virtanen M, Stansfeld SA, Fuhrer R, et al. Overtime work as a predictor of major depressive episode: a 5-year follow-up of the Whitehall II study. PLoS ONE 2012;7:e30719.

27. Gopal R, Glasheen JJ, Miyoshi TJ, et al. Burnout and internal medicine resident work-hour restrictions. Arch Intern Med 2005;165:2595-600.

28. Reed DA, Fletcher KE, Arora VM. Systematic review: association of shift length, protected sleep time, and night float with patient care, residents' health, and education. Ann Intern Med 2010;153:829-42.

29. Sargent MC, Sotile W, Sotile MO, et al. Quality of life during orthopedic training and academic practice part 1: orthopedic surgery residents and faculty. J Bone Joint Surg Am 2009;91A:2395-405.

30. Fletcher KE, Underwood W, Davis SQ, et al. Effects of work hour reduction on residents' lives-a systematic review. JAMA 2005;294:1088-100.

31. Black C, Frost D. Health at work-an independent review on sickness absence. London: The Stationary Office, 2011.

32. Waddell G, Burton K. Is work good for your health and wellbeing? London: The Stationary Office, 2006.

33. Butterworth $\mathrm{P}$, Leach LS, Strazdins L, et al. The psychosocial quality of work determines whether employment has benefits for mental health: results from a longitudinal national household panel survey. Occup Environ Med 2011;68:806-12. 
34. Lockley SW, Landrigan CP, Barger LK, et al. When policy meets physiology - the challenge of reducing resident work hours. Clin Orthop 2006:116-27.

35. ICD-10. 012 International Classification of Diseases. http://www.who. int/classifications/icd/en/ (accessed 2012).

36. van Uffelen JGZ, Wong J, Chau JY, et al. Occupational sitting and health risks: a systematic review. Am J Prev Med 2010;39: 379-88.

37. Kirsling RA, Kochar MS, Chan $\mathrm{CH}$. An evaluation of mood states among 1st-year residents. Psychol Rep 1989;65:355-66.

38. Bernard B. A critical review of epidemiologic evidence for work-related musculoskeletal disorders of the neck, upper extremity and low back. Publication No. 9741. Cincinnati: National Institute for Occupational Safety and Health, 1997.

39. Steenstra IA, Verbeek JH, Heymans MW, et al. Prognostic factors for duration of sick leave in patients sick listed with acute low back pain: a systematic review of the literature. Occup Environ Med 2005;62:851-60.

40. Ayas NT, Barger LK, Cade BE, et al. Extended work duration and the risk of self-reported percutaneous injuries in interns. JAMA 2006;296:1055-62.

41. Barger LK, Cade BE, Ayas NT, et al. Extended work shifts and the risk of motor vehicle crashes among interns. $N$ Engl $\mathrm{J} \mathrm{Med}$ 2005;352:125-34.

42. Firth-Cozens J. Emotional distress in junior house officers. BMJ 1987;295:533-6.

43. Fisman DN, Harris AD, Rubin M, et al. Fatigue increases the risk of injury from sharp devices in medical trainees: results from a case-crossover study. Infect Control Hosp Epidemiology 2007;28: 10-17.

44. Hayasaka Y, Nakamura K, Yamamoto $\mathrm{M}$, et al. Work environment and mental health status assessed by the general health questionnaire in female Japanese doctors. Ind Health 2007;45:781-6.

45. Kirkcaldy BD, Trimpop R, Cooper CL. Working hours, job stress, work satisfaction, and accident rates among medical practitioners and allied personnel. Int J Stress Manag 1997;4:79-87.

46. Rosta J, Aasland OG. Work hours and self rated health of hospital doctors in Norway and Germany. A comparative study on national samples. BMC Health Serv Res 2011;11:40.

47. Stamp T, Termuhlen P, Miller S, et al. Before and after resident work hour limitations: an objective assessment of the well-being of surgical residents. Curr Surg 2005;62:117-21.
48. Sundquist J, Johansson SE. High demand, low control, and impaired general health: working conditions in a sample of Swedish general practitioners. Scand J Public Health 2000;28:123-31.

49. Varma A, Marott J, Stoltenberg C, et al. With long hours of work, might depression then lurk? A nationwide prospective follow-up study among Danish senior medical consultants. Scand J Work Environ Health 2012;38:418-26.

50. Zahrai A, Chahal J, Stojimirovic D, et al. Quality of life and educational benefit among orthopedic surgery residents: a prospective, multicentre comparison of the night float and the standard call systems. Can J Surg 2011;54:25-32.

51. Dembe AE, Delbos R, Erickson JB. Estimates of injury risks for healthcare personnel working night shifts and long hours. Qual Saf Health Care 2009;18:336-40.

52. Lindfors PM, Nurmi KE, Meretoja OA, et al. On-call stress among Finnish anaesthetists. Anaesthesia 2006;61:859-64.

53. Selb J, Albreht T. Mortality rates of medical doctors in Slovenia in 1985 to 1999. Zdravniški vestnik 2000;(Suppl I):I47-8.

54. Lee S, McCann D, Messenger J. Working time around the world: trends in working hours, laws and policies in a global comparative perspective. Geneva, Switzerland: International Labour Office, 2007.

55. Elder A, Paterson C. Sharps injuries in UK health care: a review of injury rates, viral transmission and potential efficacy of safety devices. Occup Med (Lond) 2006;56:566-74.

56. Naghavi SHR, Sanati KA. Needlestick injuries: does left-handedness matter? Am J Infect Control 2009;37:341.

57. Connor J, Norton R, Ameratunga S, et al. Driver sleepiness and risk of serious injury to car occupants: population based case control study. BMJ 2002;324:1125-8A

58. BMA. 2013 European Working Time Directive. http://bma.org.uk/ practical-support-at-work/ewtd (accessed Dec 2013).

59. Wilson AM, Weston G. Application of airline pilots hours to junior doctors. BMJ 1989;299:779-81.

60. The European Specialist Medical Qualifications Order 1995. 1995 Statutory Instruments, 1995 No. 3208, MEDICAL PROFESSION, The European Specialist Medical Qualifications Order 1995. http:// legislation.data.gov.uk/uksi/1995/3208/made/data.htm?wrap=true (accessed Dec 2013).

61. Boorman S. NHS Health and Well-being. The Boorman Review. Leeds, 2009.

62. Williams S, Michie S, Pattani S. Improving the health of the NHS workforce. Report of the partnership on the health of the NHS Workforce. London: The Nuffield Trust, 1998. 